\title{
ANALYSIS OF ATMOSPHERIC PROPAGATION EFFECTS IN TERRASAR-X IMAGES
}

\author{
A. Danklmayer, B. Döring, M. Schwerdt
}

\author{
German Aerospace Center (DLR) \\ Microwave and Radar Institute \\ Oberpfaffenhofen, Germany
}

\author{
M. Chandra
}

\author{
Chemnitz University of Technology \\ Dept. of Microwave Eng. and Photonics \\ Chemnitz, Germany
}

\begin{abstract}
TerraSAR-X, the first civil German synthetic aperture radar (SAR) satellite has been successfully launched in 2007, June $15^{\text {th }}$. After 4.5 days the first processed image has been obtained. The overall quality of the image was outstanding, however, suspicious features could be identified which showed precipitation related signatures. These rain-cell signatures motivated a further in-depth study of the physical background of the related propagation effects. During the commissioning phase, a total of 12000 scenes have been investigated for potential propagation effects and about 100 scenes have revealed atmospheric effects to a visible extent. An interesting case of a data acquisition over New York will be presented which shows typical rain-cell signatures and the SAR image will be compared with weather-radar data acquired nearly simultaneously (within the same minute). Furthermore, in this contribution we discuss the influence of the atmosphere (troposphere) on the external calibration (XCAL) of TerraSAR-X. By acquiring simultaneous weatherradar data over the test-site and the SAR-acquisition it was possibleto improve the absolute calibration constant by 0.15 dB.
\end{abstract}

Index Terms - TerraSAR-X, SAR, Microwave imaging, Atmospheric Propagation effects, Signal Attenuation

\section{INTRODUCTION}

The purpose of this introduction is to identify, estimate and summarize the propagation effects capable of influencing space-borne synthetic aperture radar (SAR) measurements. Amongst the many effects are delays, attenuation, noise, scintillation which are caused by atmospheric gases, rain (precipitation), clouds, fog or free electrons in the ionosphere.

\section{TROPOSPHERIC EFFECTS}

The troposphere, as the lowest part of the Earth's atmosphere, reaches from the surface to approximately $12 \mathrm{~km}$ above ground and causes, amongst other effects, attenuation of traversing signals due to hydrometeors (rain, snow, hail), atmospheric gases, fog and clouds [1, 2, 3, 4]. Except at low elevation angles, the attenuation of frequencies below $1 \mathrm{GHz}$ is negligible. Insignificant contributions to the attenuation will be obtained for frequencies up to $10 \mathrm{GHz}$ due to fog and non-precipitating clouds. However the transmission spectrum exhibits peaks for frequencies around $22 \mathrm{GHz}$ and $60 \mathrm{GHz}$ due to molecular resonances from gases i.e. water vapour and oxygen. Whereas absorption effects due to atmospheric gases are present constantly and everywhere, attenuation due to condensed water in the form of precipitation, clouds and fog is infrequent and is limited to certain areas. Attenuation consists of two physical processes: the reduction of the wave's energy due to the heating of the water particles and, the scattering of energy away from the main direction of propagation.

\subsection{Attenuation through rain}

One of the major problems affecting microwave and millimetre wave bands for terrestrial and space-borne radars is the attenuation through rain $[5,6]$. Rain is the most important cause, not only due to the strong attenuation effect but also due to the fact that rain occurs most frequently. A convenient way to describe the rain intensity is the so called rainfall-rate or rain-rate given in millimetres per hour. This quantity refers to a certain flux of rain towards the surface of the Earth and may be measured by gauges on the surface. A rain rate of $4 \mathrm{~mm} / \mathrm{hr}$ is a typical value for the specification of moderate rain [5]. Typical values for the specific attenuation (attenuation for $1 \mathrm{~km}$ propagation path) of different frequency bands according to a given rain-rate are provided in [1]. In many publication an empirical relation of the form

$$
\gamma(x, t)=a \cdot R^{b}
$$

between specific attenuation $\gamma(x, t)$ and rain rate $R$ are provided $[5,7,8]$. The parameters $a$ and $b$ are dependent on the radio frequency, the raindrop size distribution, the polarization and other factors [7].

After [5], the total attenuation for a given instant of time can be obtained by integrating the specific attenuation along the path of propagation using the following expression 


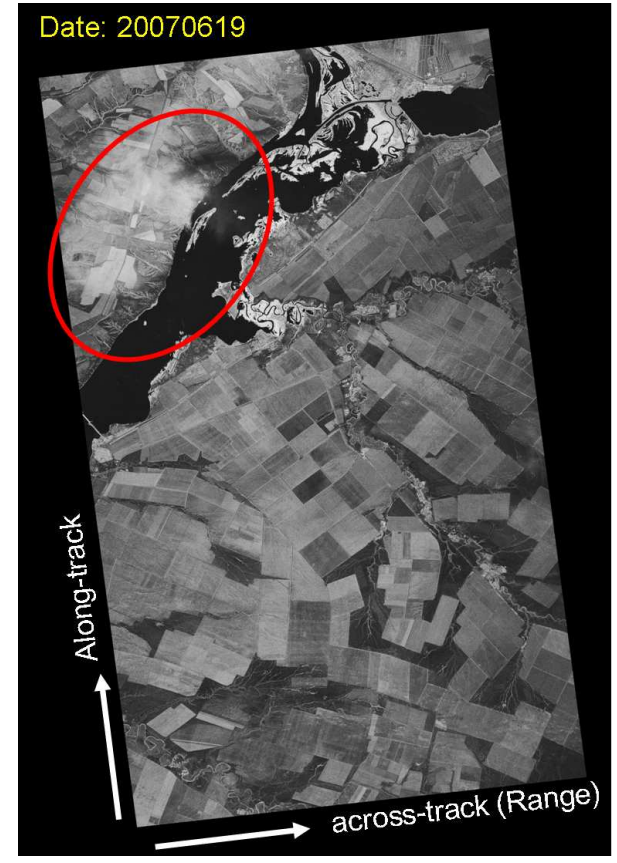

Fig. 1. An example of rain-cell-affected SAR signatures recorded with TerraSAR-X. The white shading is due to direct reflections from the rain region (shown as volume ' $A$ ' in Fig. 2). Whereas the darkly shaded areas are due to rain attenuated (blocked) signals from the ground; this effect is shown as region ' $B$ ' in the Fig. 2. The parameters of acquisition are given in Table 1.

$$
A(t)=\int_{0}^{2 h} \gamma(x, t) d x
$$

where

$$
\begin{array}{ll}
A(t) & \ldots \text { total attenuation for given time instant } \mathrm{t} \\
t & \ldots \text { time } \\
h & \ldots \text { path length } \\
\gamma(x, t) & \ldots \text { specific attenuation } \\
x & \ldots \text { position along the path of propagation }
\end{array}
$$

The specific attenuation along the slant path of propagation has to be known. However, detailed knowledge of the medium through which the signal propagates is rather limited and the temporal and spatial variation of the medium require assumptions and some modelling [3]. In the case of precipitation we may have some idea about the thermodynamic phase (ice, water, melting band) but no precise information. Using the tabulated values for the attenuation in X-band for a $2-3 \mathrm{~km}$ long path through a heavy tropical convective rain $(50 \mathrm{~mm} / \mathrm{hr}$ and more) suggests a $20-30 \mathrm{~dB}$ attenuation.
Table 1. The main acquisition parameters of the first image acquired by TerraSAR-X obtained 4.5 days after launch.

\begin{tabular}{ll}
\hline Parameter & Values \\
\hline Frequency band & X-band / 9.65 GHz \\
Image dimensions: & $\begin{array}{l}\text { Azimuth } \sim 60 \mathrm{~km} \\
\text { Range } \sim 30 \mathrm{~km}\end{array}$ \\
Location & $\begin{array}{l}50 \mathrm{~km} \text { to the west of } \\
\text { Volgograd (Russia) }\end{array}$ \\
Scene center & Lat: 48.4504 \\
& Lon: 43.5542 \\
Imaging Mode & Strip map / HH Polarization \\
\hline
\end{tabular}

\section{ANALYSIS OF DATASETS AND PHYSICAL INTERPRETATION OF ARTEFACTS IN SAR-IMAGES DUE TO PRECIPITATION}

A physical interpretation of how rain cells affect SAR images is given in Fig. 2. The image shows an imaging scenario, where the transmitted waves interact with a precipitation cell. The red rays correspond to the signals transmitted through the clouds, the black rays to the reflected ones. It can be observed, that at area 'A' which corresponds to time instant $\tau_{A}$ in the amplitude/time diagram, is due to strong backscattering from large hydrometeors. Time instant $\tau_{B}$, which corresponds to the region ' $\mathrm{B}$ ' proves that signals have been heavily attenuated. By comparing the encircled area in Fig. 1 and the amplitude/time diagram in Fig. 2, the veil in Fig. 1 corresponds to area 'A' $\left(\tau_{A}\right)$ and the shadow like black areas close to these veil in Fig. 1 corresponds to the region 'B' $\left(\tau_{B}\right)$ in Fig. 2.

These physical processes are elucidated with the help of Fig. 3 where a SAR-amplitude cutout of a cross-track profile through a rain cell is depicted. As diagrammatically shown, the backscattering is accompanied with higher amplitude values and the weak signals behind the first maxima belongs to the black shadows behind to the right hand side of the bright spots, where up to $20 \mathrm{~dB}$ difference in the $\mathrm{dB}$ level may be observed.

\section{THE INFLUENCE OF TROPOSPHERIC PROPAGATION EFFECTS ON THE CALIBRATION OF TERRASAR-X}

The influence of atmospheric effects on the external calibration of SAR systems like TerraSAR-X $(9,65 \mathrm{GHz})$ may under certain circumstances exceed the instrumental errors and there is a demand to detect and monitor extraordinary precipitation events which might occur during the data acquisition. In general the estimation of the path attenuation will be 


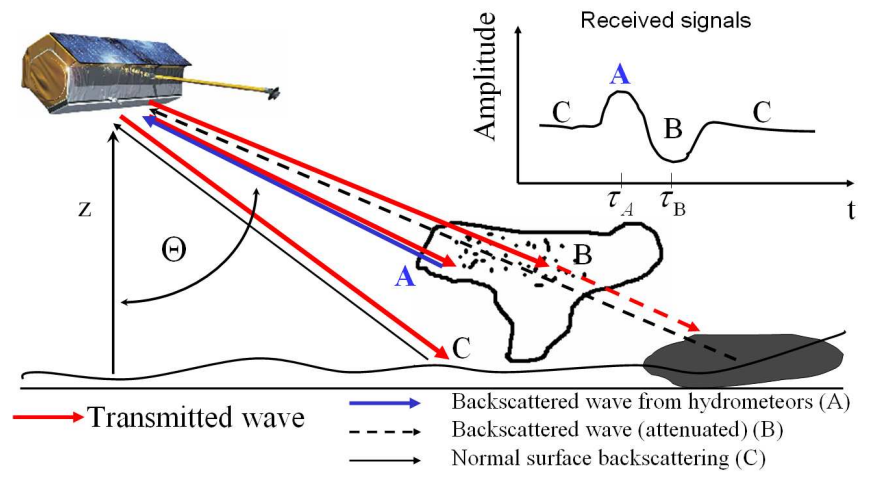

Fig. 2. A physical interpretation of rain cell signatures in SAR-images, as indicated and introduced previously in Fig. 1.

of limited accuracy and contain unremoved uncertainty. The main limitation is the resolution of the available weather data which allows not only for retrieving the path attenuation between the sensor and the calibration targets, but also phase and polarization changes. Furthermore, an insurmountable problem arises due to the fact that for each calibration target an individual radar-RHI (range height indicator) scan would be required and would have to be collected nearly simultaneously. In conclusion, there are two main problems in correcting for the attenuation due to heavy rain: Firstly, the limited accuracy of the given data in terms of the rain rate. Secondly, there are practical constraints in collecting the data, since for every external calibration target an individual RHI scan would be required, which is not feasible due the limited acquisition capability of a weather radar, i.e. it is not possible to acquire data for several different scatterer ensembles nearly simultaneously within a reasonable acquisition time. However, being able to detect high rain rates, at least, and subsequently to flag affected SAR data products, the calibration acquisitions should be monitored. Thus, flagged SAR data products can be excluded from the external calibration procedure (XCAL).

\subsection{Monitoring of the TerraSAR-X testsite during the ex- ternal calibration (XCAL) campaign}

The objective to monitor the testsite during the external calibration campaign is the detection of heavy precipitation events $(>14 \mathrm{~mm} / \mathrm{hr})$. Therefore, the weather radar data from the weather service can be utilized, which provides an update of PPIs (plan position indicator) every 15 minutes for the testsite area.

\section{Suggestion towards XCAL strategy regarding atmospheric effects}

Attenuation and possible phase changes due to heavy rain events has been identified as the main reason for erroneous propagation effects at X-band, for example, in the case of TerraSAR-X. Such events do comprise convective precipitation cells typically existent during thunderstorms.

The detection of such events can be performed using weather radar measurements. There are two different sources to estimate the rain rate. The weather radar data e.g. from the German weather service DWD and the DLR - Polarization diversity radar (POLDIRAD). The analysis of both types of data has shown good agreement as reported in [9]. Furthermore, the analysis showed that the accuracy of the data is not sufficient in terms of a deterministic correction approach in the magnitude of tenths of $\mathrm{dB}$.

The way to tackle the problem of tropospheric propagation effects due to rain is done as follows: Heavy precipitation events $(\approx 12,5 \mathrm{~mm} / \mathrm{hr}$ ) occurring approximately less than 6 hours per year will be observed and filtered using the collected weather radar data from regional weather service which allows for estimation of the rain-rate at the calibration testsite in say Southern Germany (Bavaria). As the correction of the attenuation in the magnitude of tenth $\mathrm{dB}$ is not easily implementable, affected data sets will be flagged and excluded from the XCAL, i.e. from the procedure to derive the absolute calibration factor $[9,10,11]$.

For the case of TerraSAR-X the simultanouly acquired weather radar measurents for each data take of TerraSAR-X have been thouroghly analysed. The total number of anlysed scences was 168 , where 141 measurements were acquired without any precipitation. From the remaining 27 scences with rain, 4 measurements could be identified with strong rain and they have been in turn excluded from the procedure to derive the averaged absolute calibration constant. Thus the overall improvement of the absolute calibration constant was $0.15 \mathrm{~dB}$.

\section{CONCLUSIONS AND OUTLOOK}

The conclusions in this contribution are manifold. First of all, propagation effects can be very important and they need to be considered in interpreting radar images. Attenuation caused by heavy rain events, has been identified as the main potential reason for image degradation and artefacts. The underlying analysis comprised more than 12000 data takes of TerraSAR$\mathrm{X}$. Maximum attenuation up to $20 \mathrm{~dB}$ through the precipitation volumes may occur in the cases of heavy precipitation, for instance, for data takes acquired over the Brazilian rainforest. Clouds with low liquid-water content or low rain rates and homogenous distribution will cause no or negligible distortions (visible artefacts). The flexible TerraSAR-X instrument offers new possibilities for investigation of propagation effects in SAR-imaging and the results presented herein may be also important for future systems operating with higher nominal frequencies, e.g. the Ku-band where propagation effects due to precipitation will become even more severe. For such cases the results presented in this contribution may act 


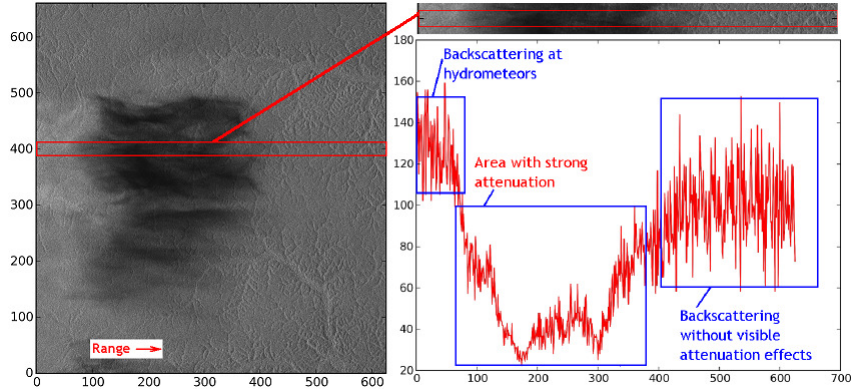

Fig. 3. A depiction of a slant range reflectivity profile ("Asope") for the rain cell cut from a very recent TerraSAR-X measurement.

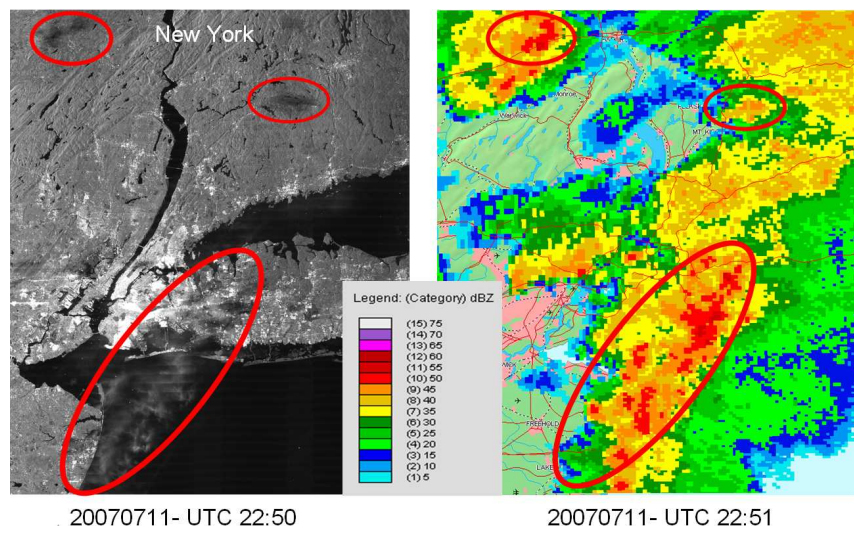

Fig. 4. A test-case showing a comparison of SAR and weatherradar data acquired nearly simultaneously (within the same minute) over New York, US. A good agreement between the rain-cell signatures in the SAR-image (left hand side) and the weatherradar image (right hand side) can be observed. The effects are most pronounced for reflectivieties of up to $50 \mathrm{dBz}$.

as a useful reference. In a test-case, it was shown for the first time that simultaneous precipitation measurements with weather radars are in good agreement with the observed raincell signature observed in the SAR-images and that such simultaneous measurements that are now available for further in-depth study of attenuation effects are an invaluable source of information for further investigations of attenuation effects and their quantification.

\section{REFERENCES}

[1] ITU, Recommendation, propagation in non-ionized media, 1994.

[2] M. P. Hall, Effects of the Troposphere on radio communication, IEE, 1979.

[3] A. W. Doerry, "Atmospheric loss considerations for syn-

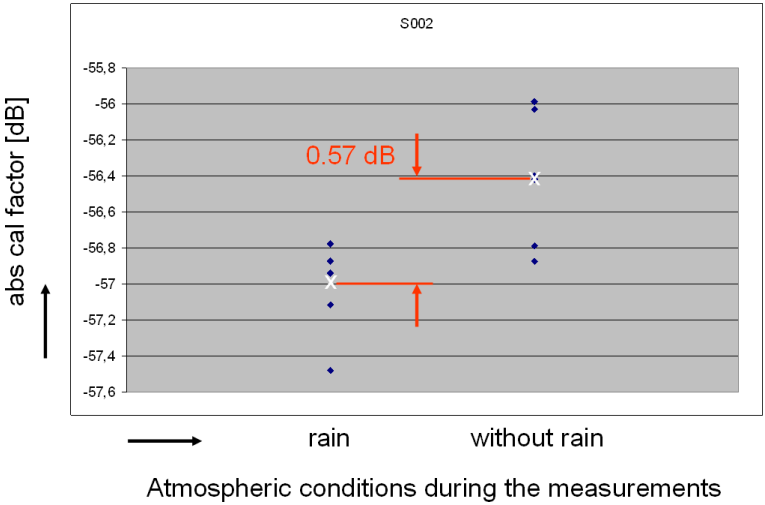

Fig. 5. The diagram shows the difference in the absolute calibration factor for measurements taken at different atmospheric condition. The white ' $x$ ' represents the mean value of measurements of the passive targets (corner reflectors). A difference of $0.57 \mathrm{~dB}$ has been observed for measurements during rain conditions and conditions without any rain.

thetic aperture radar design and operation," in Proceedings of SPIE, 2004.

[4] T. Oguchi, "Electromagnetic Wave Propagation and Scattering in Rain and Other Hydormeteors," Proceedings of the IEEE, vol. 9, pp. 1029-1079, Sepember 1983.

[5] R. K. Crane, Electromagnetic Wave Propagation Through Rain, John Wiley and Sons, 1996.

[6] P. Ferrazzoli and G. Schiavon, "Rain-induced modification of SAR performance," Adv. Space Res., vol. 7, no. 11, pp. 269-272, 1987.

[7] R. L. Olsen, D. V. Rogers, and D. B. Hodge, "The $a \cdot R^{b}$ relation in calculation of rain attenuation," IEEE Transactions on antennas and propagation, vol. 26, no. 2, pp. 318-329, 1978.

[8] V. N. Bringi and V. Chandrasekar, Polarimetric Doppler Weather Radar, Principles and Applications, Cambridge University Press, New York, 2001.

[9] A. Danklmayer, "Analysis of propagation effects during the external calibration," Tech. Rep. TX-IOCS-TN-PN4312, DLR, 2006, Available through special permission.

[10] Marco Schwerdt, David Hounam, José Luis AlvarezPérez, and Thomas Molkenthin, "The Calibration Concept of TerraSAR-X, a Multiple Mode High Resolution SAR," Vol. 31, no.1, Canadian Journal of Remote Sensing, Feb 2005.

[11] M. Schwerdt, B. Bräutigam, M. Bachmann, and B. Döring, "Efficient calibration and first results of TerraSAR-X," in Advanced SAR Workshop, 2007. 